\title{
Korean physicians' attitudes toward the prenatal screening for fetal aneuploidy and implementation of non-invasive prenatal testing with cell-free fetal DNA
}

\author{
Soo Hyun Kim', Kun Woo Kim², You Jung Han ${ }^{3}$, Seung Mi Lee ${ }^{4}$, Mi-Young Lee, Jae-Yoon Shim5, Geum Joon Cho ${ }^{6}$, Joon Ho Lee, \\ Soo-young $\mathrm{Oh}^{8}$, Han-Sung Kwon ${ }^{9}$, Dong Hyun Cha, ${ }^{1, *}$ and Hyun Mee Ryu ${ }^{3, *}$ \\ 'Department of Obstetrics and Gynecology, CHA Gangnam Medical Center, CHA University School of Medicine, Seoul, Korea \\ ${ }^{2}$ Department of Obstetrics and Gynecology, Hamchoon Women's Clinic, Seoul, Korea \\ ${ }^{3}$ Department of Obstetrics and Gynecology, Cheil General Hospital and Women's Healthcare Center, Dankook University College of Medicine, \\ Seoul, Korea \\ ${ }^{4}$ Department of Obstetrics and Gynecology, Seoul Metropolitan Government Seoul National University Boramae Medical Center, Seoul National \\ University College of Medicine, Seoul, Korea \\ ${ }^{5}$ Department of Obstetrics and Gynecology, Asan Medical Center, University of Ulsan College of Medicine, Seoul, Korea \\ ${ }^{6}$ Department of Obstetrics and Gynecology, Korea University Guro Hospital, Seoul, Korea \\ ${ }^{7}$ Department of Obstetrics and Gynecology, Severance Hospital, Yonsei University Health System, Seoul, Korea \\ ${ }^{8}$ Department of Obstetrics and Gynecology, Samsung Medical Center, Sungkyunkwan University School of Medicine, Seoul, Korea \\ ${ }^{9}$ Department of Obstetrics and Gynecology, Konkuk University Medical Center, Konkuk University School of Medicine, Seoul, Korea
}

\begin{abstract}
Purpose: Physicians' attitudes may have a strong influence on women's decision regarding prenatal screening options. The aim of this study is to assess the physicians' attitudes toward prenatal screening for fetal aneuploidy including non-invasive prenatal testing (NIPT) in South Korea.

Materials and Methods: Questionnaires were distributed and collected at several obstetrics-gynecological conferences and meetings. The questionnaire included 31 multiple choice and 5 fill-in-the-blank questions. Seven questions requested physicians' demographic information, 17 questions requested information about the NIPT with cell-free fetal DNA, and 12 questions requested information about general prenatal screening practices.

Results: Of the 203 obstetricians that completed the survey. In contrast with professional guidelines recommending the universal offering of aneuploidy screening, only $53.7 \%$ answered that prenatal aneuploidy testing (screening and/or invasive diagnostic testing) should be offered to all pregnant women. Physicians tended to have positive attitudes toward the clinical application of NIPT as both primary and secondary screening methods for patients at high-risk for fetal trisomy. However, for patients at average-risk for fetal trisomy, physicians tended to have positive attitudes only as a secondary screening method. Physicians with more knowledge about NIPT were found to tend to inform their patients that the detection rate of NIPT is higher.

Conclusion: This is the first study to investigate expert opinion on prenatal screening in South Korea. Education of physicians is essential to ensure responsible patient counseling, informed consent, and appropriate management after NIPT.
\end{abstract}

Key words: Physicians' practice patterns, Prenatal diagnosis, Genetic testing, Prenatal care.

Received: 2 November 2018, Revised: 13 December 2018, Accepted: 13 December 2018, Published: 31 December 2018

${ }^{*}$ Co-corresponding author: Dong Hyun Cha M.D., Ph.D. iD http://orcid.org/0000-0003-0722-1714

Department of Obstetrics and Gynecology, CHA Gangnam Medical Center, CHA University School of Medicine, 566 Nonhyun-ro, Gangnam-gu, Seoul 06135, Korea.

Tel: +82-2-3468-3314, Fax: +82-2-558-1112, E-mail: chadh001@chamc.co.kr

Hyun Mee Ryu, M.D., Ph.D. (iD http://orcid.org/0000-0002-0710-2582

Department of Obstetrics and Gynecology, Cheil General Hospital and Women's Healthcare Center, Dankook University College of Medicine, 17 Seoae-ro 1-gil, Jung-gu, Seoul 04619, Korea.

Tel: +82-2-2000-7175, Fax: +82-2-2000-7793, E-mail: hmryu2012@naver.com

Conflict of interest: The authors declare that they do not have any conflicts of interest.

(C) This is an open-access article distributed under the terms of the Creative Commons Attribution Non-Commercial License (http://creativecommons.org/licenses/by-nc/4.0/) which permits unrestricted non-commercial use, distribution, and reproduction in any medium, provided the original work is properly cited.

(c) Copyright 2018 by the Korean Society of Medical Genetics and Genomics 


\section{Introduction}

As cell-free fetal DNA in maternal blood is available for prenatal screening, physicians can offer various prenatal screening options to their patients $[1,2]$. Test selections may be affected by a variety of factors, such as the country's culture, its health system, its abortion laws, the costs, woman's prior risk, and the information provided by the test, as well as parental level of awareness [3-5]. Physicians' attitudes may have a strong influence on pregnant women's decision to consent to a prenatal screening [6-8]. The American College of Obstetricians and Gynecologists recommends that all pregnant women should be offered the option of aneuploidy screening or diagnostic testing for fetal genetic disorders, regardless of maternal age [9]. Maternal serum screening has been covered by the national health insurance system, and prenatal genetic screening is performed in more than $95 \%$ of all pregnancies in South Korea. This screening usually begins in the first trimesters.

Non-invasive prenatal testing (NIPT) using cell-free fetal DNA shows the highest sensitivity and specificity for the screening of Down syndrome in high risk pregnancies and enables prenatal screening as early as 9 weeks of gestation. Commercialized NIPT is widely used in many countries. The reported detection rate and false positive rate for Down syndrome are more than 98\% and less than $0.5 \%$, respectively $[10,11]$. Because of this low false positive rate, it is assumed that the implementation of NIPT as a screening may reduce the number of invasive procedures.

With the emergence of NIPT offering, healthcare providers are challenged to keep abreast of the underlying technology, test performance, and clinical utility of these new options. As physicians are obligated to help their patients in informed decision making, their understanding and attitudes about NIPT and plans for clinical use are of significant interest.

This study aims to survey obstetrician-gynecologists about their current screening practices, their attitudes about which of their prenatal patients they were most likely to offer NIPT to, and how they would use this information in the landscape of prenatal testing options for aneuploidy. It is important to know the opinions of health professionals to further develop NIPT testing system in South Korea.

\section{Materials and Methods}

We set out to understand the knowledge base and opinions of obstetrician-gynecologists about NIPT in South Korea, through an incentive-based offline survey. The survey was distributed and collected at several obstetrics-gynecological conferences and meetings from September 2016 to May 2017. All participants were completely trained and beyond their residency. The questionnaire included items regarding prenatal screening for fetal aneuploidy and implementation of NIPT in practice. The present study was reviewed and approved by the medical ethical committee of Dankook University (IRB no. CGH-IRB-2016-13). Participants were asked to provide verbal consent to participate in the study at the time of recruitment, and completion and return of the questionnaires signifies implied consent.

The survey questionnaire was developed based on a similar survey and tested with eleven obstetricians prior to the study (Supplementary material 1) [12]. After the pilot test, the questionnaire required only minor grammatical changes.

There were 31 multiple choice and 5 fill-in-the-blank questions. Seven questions requested physicians' demographic information, 17 questions requested information about the NIPT, and 12 questions requested information about general prenatal screening practices. The survey used a 5 -point Likert scale as follows: 1 , completely disagree; 2 , disagree; 3 , neither agree nor disagree; 4, agree; 5, completely agree. Likert scales were analyzed as continuous variables. Multiple responses were allowed for in some categorical data, and ranking scores were collected and analyzed separately. The data were transferred to an Excel spreadsheet (Microsoft Corp., Redmond, WA, USA) and analyzed using the IBM SPSS Statistics software, version 23.0 (SPSS Inc., Chicago, IL, USA). Data analysis yielded frequency distributions and percentages for each of the survey questions. Using descriptive statistics, continuous variables were presented as mean \pm standard deviation and categorical data as frequency (\%) for each of the survey questions.

\section{Results}

A total of 203 questionnaires were analyzed. Not all participants answered all items. All respondents were included in the analysis, but non-respondents in each question were excluded from analysis. Females comprised $67 \%$ of respondents. Respondents spanned a wide range of ages with a mean of $41.5 \pm 8.1$ years of age: $26.7 \%$ (54/202) were 35 years old or younger, $44.1 \%$ (89/202) were 36 to 45 years old, 22.3\% (45/202) were 46 to 55 years old, and 6.9\% (14/202) were 56 to 65 years old. The average male age was higher than the average female age ( 47.5 vs. 43.5 years of age). Ninty-four (46.3\%) respondents have practiced for over 11 years; while $36.5 \%$ of those surveyed practiced as obstetricians for less than 5 years. Eighty-eight of 
203 (43.3\%) completed a fellowship in maternal-fetal medicine. Those surveyed practiced in a variety of settings, including $52.7 \%$ in university hospital or academic clinic, $29.6 \%$ in private clinic with delivery room. The demographic characteristics of the respondents are shown in Table 1.

About 93\% (187/201) of respondents identified a subset of their patients as being at high-risk for fetal aneuploidy. Table 2 shows the criteria for the identification of patients as high risk for aneuploidy. A total of 53.7\% (108/201) answered that prenatal aneuploidy testing (screening and/or invasive diagnostic testing) should be offered to all pregnant women, whereas

Table 1. The demographic characteristics of the respondents $(n=203)$

\begin{tabular}{lc}
\hline \multicolumn{1}{c}{ Characteristic } & Value \\
\hline Gender & $136(67.0)$ \\
\hline Female & $67(33.0)$ \\
\hline Male & \\
Clinical experience (yr) & $74(36.5)$ \\
$\leq 5$ & $35(17.2)$ \\
$6-10$ & $41(20.2)$ \\
$11-15$ & $29(14.3)$ \\
$16-20$ & $24(11.8)$ \\
\hline 221 & \\
Fellowship & $88(43.3)$ \\
Maternal-fetal medicine & $39(19.2)$ \\
Other specialty & $76(37.4)$ \\
None & \\
Practice setting & $107(52.7)$ \\
\hline University hospital & $20(9.9)$ \\
General hospital & $60(29.6)$ \\
Private clinic with delivery room & $16(7.9)$ \\
Private clinic without delivery room & $149(73.4)$ \\
Experience using non-invasive prenatal testing & $39(19.2)$ \\
Yes & $15(7.4)$ \\
\hline No & \\
\hline No response & \\
\hline
\end{tabular}

Values are presented as number (\%).

Table 2. Criteria and responses for the identification of patients as high risk for aneuploidy

\begin{tabular}{lr}
\hline $\begin{array}{l}\text { Which of the following criteria do you use to determine that } \\
\text { a patient is at high risk for carrying an aneuploid pregnancy? } \\
\text { (Check all that apply) }\end{array}$ \\
\hline \multicolumn{2}{c}{ Positive maternal serum screening result } \\
Maternal age & 84.0 \\
Ultrasound findings suggestive of aneuploidy & 83.5 \\
Family history of aneuploidy & 83.5 \\
Previous pregnancy affected by aneuploidy & 79.0 \\
Other & 70.5 \\
\hline
\end{tabular}

$46.3 \%$ answered that aneuploidy testing should be offered to only high-risk pregnant women.

The stated top three advantages of the conventional screening tests were as follows: (1) safe for the fetus, (2) long history and experience with the test, (3) covered by insurance. The top three disadvantages of the conventional screening test were as follows: (1) high false positive, (2) patient anxiety related to false positive results, (3) inconsistency between nuchal translucency ultrasound and the test (Fig. 1). The stated top three advantages of NIPT were as follows: (1) safe for the fetus, (2) available in the first trimester, (3) high detection rate. The top three disadvantages of NIPT were as follows: (1) should confirm results using

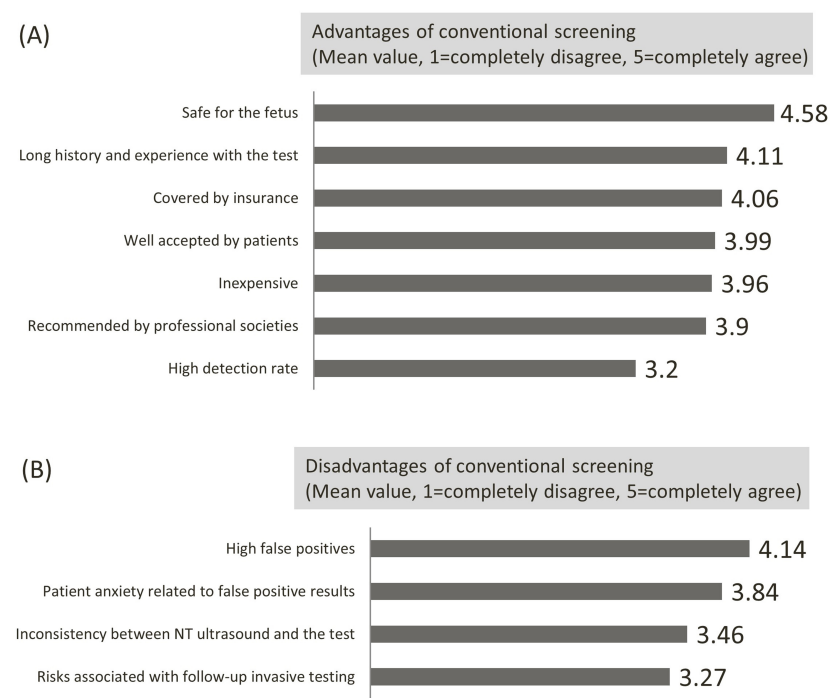

Fig. 1. Advantages and disadvantages of conventional screening tests. NT, nuchal translucency.

(A)

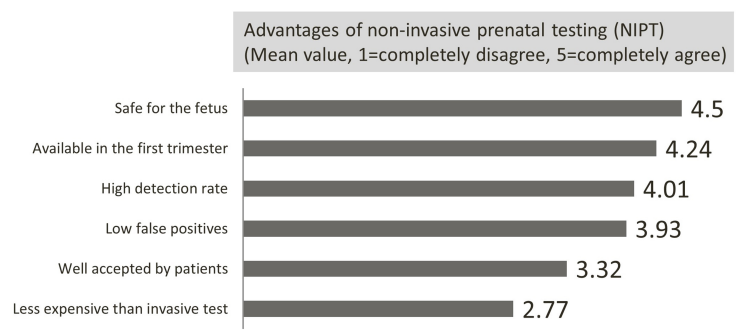

(B)

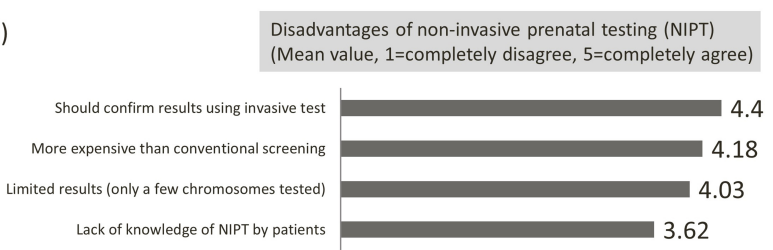

Fig. 2. Advantages and disadvantages of non-invasive prenatal testing. 
invasive test, (2) more expensive than conventional screening, (3) limited results (Fig. 2).

Physicians were asked a series of questions about the type of tests they currently provide. A total of 68.9\% (135/196) indicated that they provide integrated test followed by quadruple test $(20.9 \%, 41 / 196)$ for detection of aneuploidy in singletone pregnancies. In multiple pregnancies, 55.2\% (107/194) indicated that they are providing integrated test followed by quadruple test $(34.0 \%, 66 / 194)$. The questions above allowed multiple choices (Table 3).

Regarding adoption of new technologies, 33.5\% of respondents established their standard practice flow of prenatal test

Table 3. The type of maternal serum screening tests which they currently provide (multiple choices)

\begin{tabular}{|cc}
\hline \multicolumn{1}{c}{ Screening test } & Value \\
\hline Singletone pregnancy $(\mathrm{n}=196)$ & \\
\hline Integrated & $135(68.9)$ \\
\hline Quadruple & $41(20.9)$ \\
Sequential & $27(13.8)$ \\
Combined & $3(1.5)$ \\
\hline Triple & $2(1.0)$ \\
Multiple pregnancy $(\mathrm{n}=194)$ & \\
\hline Integrated & $107(55.2)$ \\
\hline Quadruple & $66(34.0)$ \\
Sequential & $25(12.9)$ \\
Combined & $4(2.1)$ \\
Triple & $2(1.0)$ \\
\hline
\end{tabular}

Values are presented as number (\%).

Table 4. The length of time to inform/counsel their patients about prenatal tests for aneuploidy $(n=196)$

\begin{tabular}{|cc}
\hline \multicolumn{1}{c}{ The length of time } & Value \\
\hline The general explanation (min) & $10(5.1)$ \\
$<1$ & $56(28.6)$ \\
$1-3$ & $73(37.2)$ \\
$4-5$ & $31(15.8)$ \\
$6-7$ & $18(9.2)$ \\
$8-10$ & $8(4.1)$ \\
$>10$ & \\
If there are positive test results (min) & $2(1.0)$ \\
$<1$ & $10(5.1)$ \\
$1-3$ & $34(17.3)$ \\
$4-5$ & $47(24.0)$ \\
$6-7$ & $58(29.6)$ \\
$8-10$ & $45(23.0)$ \\
\hline 10
\end{tabular}

Values are presented as number (\%). according to the individual physician's own choice. A total of $32.5 \%$ established it according to informal consensus among physicians based on group discussions and 19.6\% established it according to policy by society's guidelines.

A total of $53.8 \%$ (106/197) informed their patients about prenatal tests for aneuploidy between 8 and 10 weeks of gestation and 28.9\% (57/197) informed their patients between 11 and 13 weeks of gestation. Table 4 shows the length of time to inform or counsel their patients about prenatal tests for aneuploidy. The general explanation of prenatal tests for aneuploidy took more than 4 minutes in $66.3 \%$. If there are positive test results, $52.6 \%$ of physicians took more than 8 minutes to counsel patients. About 73.4\% had experience using NIPT in their practice. A total of $67.2 \%$ used their own pre-prepared explanatory notes or brochures about prenatal test or NIPT.

Physicians were asked to check all the items of information of pretest and posttest counseling about NIPT that they already knew [13]. We made an awareness index (Al) which was the total number of checked items of information by each respondent. The range of Al was from 1 to 13. All the items are included in supplementary file. Physicians previously knew $9.3 \pm 3.9$ items of information. About $88.2 \%$ knew that there is a possibility of false-positive screening results, necessitating recommendation of confirmative testing (chorionic villous sampling or amniocentesis). A total of 55.9\% knew that risk assessment of NIPT is

Table 5. Acceptance of the clinical application of NIPT in each of situations for testing patients at high-risk for fetal trisomy ${ }^{a}$

\begin{tabular}{lccc}
\hline & Agree & Unsure & Disagree \\
\hline Primary screening in all high-risk patients (as sole test) $(\mathrm{n}=175)$ \\
\hline Total & $76(43.4)$ & $56(32.0)$ & $43(24.6)$ \\
High Al & $51(44.3)$ & $38(33.0)$ & $26(22.6)$ \\
Medium Al & $8(30.8)$ & $9(34.6)$ & $9(34.6)$ \\
Low Al & $17(50.0)$ & $9(26.5)$ & $8(23.5)$ \\
Primary screening in all high-risk patients along with ultrasound $(\mathrm{n}=181)$ \\
Total & $125(69.1)$ & $42(23.2)$ & $14(7.7)$ \\
High Al & $85(72.6)$ & $26(22.2)$ & $6(5.1)$ \\
Medium Al & $19(65.5)$ & $6(20.7)$ & $4(13.8)$ \\
Low Al & $21(60.0)$ & $10(28.6)$ & $4(11.4)$
\end{tabular}

Secondary screening (abnormal ultrasound or positive serum screening result) $(n=174)$

$\begin{array}{lrrc}\text { Total } & 106(60.9) & 38(21.8) & 30(17.2) \\ \text { High Al } & 66(58.4) & 23(20.4) & 24(21.2) \\ \text { Medium Al } & 16(61.5) & 8(30.8) & 2(7.7) \\ \text { Low Al } & 24(68.6) & 7(20.0) & 4(11.4)\end{array}$

Values are presented as number (\%).

NIPT, non-invasive prenatal testing; Al, awareness index.

${ }^{a}$ Al which was the total number of checked items of information about NIPT by each respondent. 
less dependent on gestational age. We divided respondents into 3 groups according to Al value. High Al group consisted of those with an Al of 10 or more. Low Al group is composed of those with an Al of 5 or less $(21.5 \%, 42 / 195)$. A total of $62.6 \%$ of those surveyed were in high Al group (122/195).

Table 5 shows that physicians in high and low Al groups tended to have positive attitudes toward the clinical application of NIPT as both primary and secondary screening methods for patients at high-risk for fetal trisomy. There was no difference among three Al groups. A total of 60.9\% (106/174) agreed it as a secondary screening method in high-risk patients. A total of $69.1 \%$ agreed it as a primary screening method along with ultrasound and $43.4 \%$ agreed it as a sole primary screening method in high-risk patients. However, for patients at averagerisk for fetal trisomy, physicians tended to have positive attitudes toward the clinical application of NIPT only as a secondary screening method (65.4\% agreed; $20.7 \%$ were unsure; $14.0 \%$ disagreed). About $25.6 \%$ agreed it as a primary screening method along with ultrasound in average-risk patients (32.4\% were unsure; $33.5 \%$ disagreed). Only $18.5 \%$ agreed it as a sole primary screening method in average-risk patients (35.7\% were unsure; $45.8 \%$ disagreed). In subgroup analysis, physicians within the low Al group tended to have positive attitudes toward the clinical application of NIPT as a primary screening method even

Table 6. Acceptance of the clinical application of NIPT in each of situations for testing patients at average-risk for fetal trisomy ${ }^{a}$

\begin{tabular}{lccr}
\hline & Agree & Unsure & Disagree \\
\hline Primary screening in all high-risk patients (as sole test) $(\mathrm{n}=168)$ \\
\hline Total & $31(18.5)$ & $60(35.7)$ & $77(45.8)$ \\
High Al & $18(16.2)$ & $36(32.4)$ & $57(51.4)$ \\
Medium Al & $2(8.0)$ & $12(48.0)$ & $11(44.0)$ \\
Low Al & $11(34.4)$ & $12(37.5)$ & $9(28.1)$ \\
Primary screening in all high-risk patients along with ultrasound ( $\mathrm{n}=176)$ \\
Total & $57(32.4)$ & $60(34.1)$ & $59(33.5)$ \\
High Al & $32(27.8)$ & $38(33.0)$ & $45(39.1)$ \\
Medium Al & $7(26.9)$ & $11(42.3)$ & $8(30.8)$ \\
Low Al & $18(51.4)$ & $11(31.4)$ & $6(17.1)$
\end{tabular}

Secondary screening (abnormal ultrasound or positive serum screening result) $(n=179)$

\begin{tabular}{lccc} 
Total & $117(65.4)$ & $37(20.7)$ & $25(14.0)$ \\
High Al & $75(64.7)$ & $20(17.2)$ & $21(18.1)$ \\
Medium Al & $18(64.3)$ & $9(32.1)$ & $1(3.6)$ \\
Low Al & $24(68.6)$ & $8(22.9)$ & $3(8.6)$ \\
\hline
\end{tabular}

Values are presented as number (\%).

NIPT, non-invasive prenatal testing; Al, awareness index.

${ }^{a}$ Al which was the total number of checked items of information about NIPT by each respondent. when their patients are at average-risk for fetal trisomy (Table 6).

A total of $72.3 \%$ of physicians informed their patients that the detection rate of NIPT is 98 or $99 \%$. The number of respondents who answered both the question about the detection rate of NIPT and the one about NIPT awareness was 186. The AI of physicians who inform their patients of the detection rate of NIPT as $99 \%$ is $10.05 \pm 3.45(n=99)(100 \%, 9.67 \pm 1.53, n=3$; $98 \%, 9.65 \pm 3.99, n=46 ; 97 \%, 7.13 \pm 3.98, n=16 ; 96 \%, 7.32 \pm 4.36$, $\mathrm{n}=22$ ). Physicians with a high Al value were found to tend to inform their patients that the detection rate of NIPT is high. But, it was not statistically significant. Al was not correlated with age, fellowship, practice setting, clinical experience nor the average number of deliveries that they have.

The survey asked a fill-in-the-blank question about the positive predictive value (PPV) of NIPT that physicians inform their patients of. It shows a wide range between $1 \%$ and $100 \%$ (75.8 $\pm 28.5 \%)$. A total of 170 respondents answered both the question about PPV and that of NIPT awareness (Fig. 3). Five of these respondents answered that PPV could be different in a certain trisomy.

About $50.8 \%$ of respondents (101/199) thought that their patients were interested in NIPT and 28.6\% thought that they were neutral about NIPT.

A total of 188 physicians answered that they could recommend NIPT to their patients as the dominant primary screening strategy over conventional screening if the cost of NIPT would be $285,900 \pm 144,500$ won (around $270 \pm 135$ USD). $40.3 \%$ (79/196) were positive about the clinical relevance of prenatal screening for sex chromosomes using NIPT. About $64.6 \%$

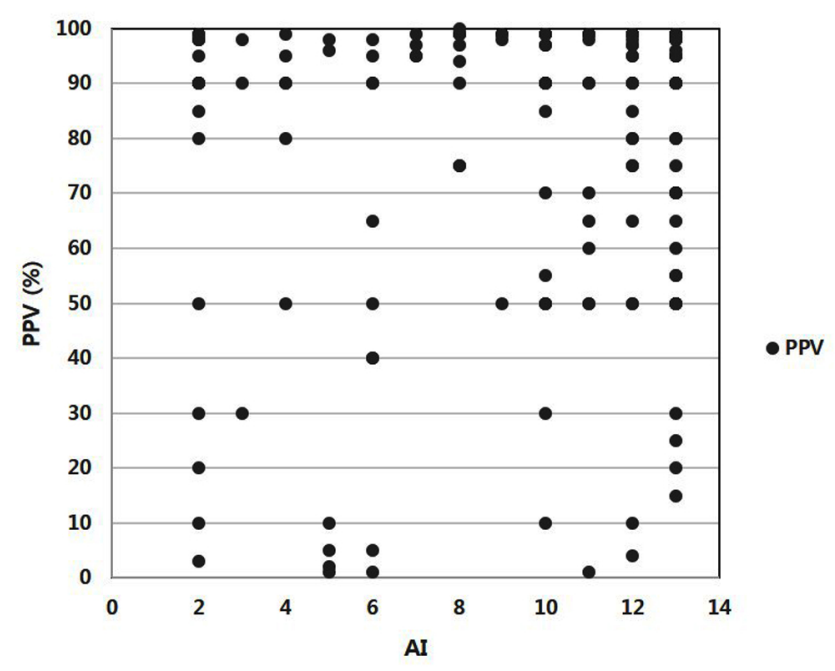

Fig. 3. Positive predictive value (PPV) of non-invasive prenatal testing which physicians inform their patients of and awareness index (Al). 
(126/195) were positive about the clinical relevance of prenatal screening for microdeletions using NIPT.

A total of $54.9 \%$ of respondents answered that they usually get information about NIPT through attending lectures or conferences. Also, 31.3\% search through journals and articles and $30.8 \%$ get information through professional society's recommendations or guidelines.

\section{Discussion}

This study was designed to ascertain the physicians' attitudes toward prenatal screening for fetal aneuploidy including NIPT in South Korea. Most obstetricians had positive attitudes toward the clinical application of NIPT as a secondary screening method. As for the primary screening, the majority agreed that it should be performed only in high-risk patients. Most physicians had positive attitudes towards the clinical application of prenatal screening for microdeletions. Result showing that physicians with less knowledge about NIPT had more positive attitudes toward the clinical application of NIPT is interesting. There was a discrepancy between high Al group and low Al group in acceptance of NIPT as primary screening test for patients at averagerisk for fetal trisomy. However, there was no substantial discrepancy between high Al group and low Al group in acceptance of NIPT for patients at high risk for fetal trisomy. The cause of this tendency is unknown, but one of possible reason of this could be that physicians with less knowledge might accept NIPT more easily as they do not fully understand the limitation of NIPT. Only $60.5 \%$ of respondents knew that uninformative test result can be reported. A total of $63.1 \%$ knew that there's still potential risk of chromosomal abnormalities even when the NIPT result is negative.

The high detection rate for NIPT is often highlighted by laboratory testing materials rather than focusing on the individual patient's PPV. Some physicians might have difficulty keeping abreast of new technology and screening strategy. This may confuse physicians and therefore PPV of NIPT, which is informed to patients by physicians, shows a wide range between $1 \%$ and $100 \%$.

The time that two thirds of survey respondents spent to inform their patients about prenatal tests for aneuploidy was between 1 and 5 minutes. If there were positive test results from prenatal screening, 52.6\% answered that it took over 8 minutes to counsel patients. Time mentioned above counted the consultation time by physicians only, and not the nurse-counseling time. One national survey reported that $45.8 \%$ of respondents answered that about 15 minutes is appropriate amount of time for pretest counseling [14]. Our survey shows that amount of time for pretest counseling by physician was shorter compared to above ideal time. This might be affected by medical policy circumstance in South Korea, which is quite different from other countries' policies. The National Health Insurance System covers many medical costs and due to this policy, patients can visit medical clinics easier and more frequently in South Korea. But number of physicians per 1,000 populations is similar to Japan, USA or Canada's number, which of reported by World Health Organization's Global Health Workforce Statistics [15]. Therefore, physicians should see a lot more patients within a certain time. Because of this, pre-prepared explanatory notes or brochures explaining about prenatal screening test are frequently used (67.2\% of participants), and this is thought to be helpful. Also, making video clips explaining about prenatal screening and sending them to patients via SNS or uploading on internet might be another supportive option.

A significant percentage $(46.3 \%, 93 / 201)$ felt that screening should be offered only to high-risk patients in contrast with professional guidelines recommending the universal offering of aneuploidy screening [9]. It is significantly different from US physicians' attitudes (approximately 12\%) [12]. Korean physicians' attitude also might be affected by special medical policy circumstance in South Korea. Maternal serum marker screening has been covered by the national health insurance system and public health center services quadruple screening test for free. Prenatal genetic screening is performed in more than 95\% of all pregnancies. Whereas abortion in South Korea is illegal in most circumstances even if the fetus has a lethal anomaly [16]. This contradictory medical environment could be such a weigh on physicians' minds. Therefore, despite professional guidelines, some individual practitioner beliefs are in conflict with their clinical practices.

The difference of physicians' attitudes could lead to several problems. If the NIPT test is conducted without adequate pretest and posttest counseling, the test results might thus create misunderstanding or misinterpretation as well as increase of medical costs. Although the survey did not intend to give information about pretest and posttest counseling regarding NIPT to physicians, those who participated this survey might have acquired knowledge about them through this survey.

The respondents were obstetrician-gynecologists who attended at obstetrics and gynecology conferences or meetings. Physicians included in this survey are thought to be potentially motivated by an interest of NIPT and they might be eager to 
learn new technologies and clinical strategies compared to physicians who did not have chance to go through this survey. Therefore, sampling bias cannot be excluded.

This is the first study to investigate expert opinion on prenatal screening in Korea. Based on these findings, education and reeducation of physicians is essential to ensure responsible patient counseling, informed consent, and appropriate management after NIPT. Attending lectures or conferences would be another preferred option.

\section{Acknowledgements}

This research was supported by a grant of the Korea Health Technology R\&D Project through the Korea Health Industry Development Institute (KHIDI), funded by the Ministry of Health \&t Welfare, Republic of Korea (grant number : HC15C1336). We thank all physicians who participated and responded in the survey.

\section{Supplementary Material}

Supplementary Material can be found via https://doi. org/10.5734/JGM.2018.15.2.72

\section{References}

1. Chiu RW, Akolekar R, Zheng YW, Leung TY, Sun $H$, Chan KC, et al. Non-invasive prenatal assessment of trisomy 21 by multiplexed maternal plasma DNA sequencing: large scale validity study. BMJ 2011; 342:c7401.

2. Bianchi DW, Platt LD, Goldberg JD, Abuhamad AZ, Sehnert AJ, Rava RP; MatErnal BLood IS Source to Accurately diagnose fetal aneuploidy (MELISSA) Study Group. Genome-wide fetal aneuploidy detection by maternal plasma DNA sequencing. Obstet Gynecol 2012;119:890901.

3. Dahl K, Kesmodel U, Hvidman L, Olesen F. Informed consent: attitudes, knowledge and information concerning prenatal examinations. Acta Obstet Gynecol Scand 2006;85:1414-9.

4. van den Berg $M$, Timmermans DR, ten Kate $L P$, van Vugt JM, van der Wal G. Informed decision making in the context of prenatal screening. Patient Educ Couns 2006;63:110-7.
5. van den Heuvel A, Marteau TM. Cultural variation in values attached to informed choice in the context of prenatal diagnosis. Semin Fetal Neonatal Med 2008;13:99-102.

6. Dormandy E, Marteau TM. Uptake of a prenatal screening test: the role of healthcare professionals' attitudes towards the test. Prenat Diagn 2004;24:864-8.

7. Lewis SM, Cullinane FM, Carlin JB, Halliday JL. Women's and health professionals' preferences for prenatal testing for Down syndrome in Australia. Aust N Z J Obstet Gynaecol 2006;46:205-11.

8. Hall S, Chitty L, Dormandy E, Hollywood A, Wildschut HI, Fortuny A, et al. Undergoing prenatal screening for Down's syndrome: presentation of choice and information in Europe and Asia. Eur J Hum Genet 2007;15:563-9.

9. Committee on Practice Bulletins-Obstetrics, Committee on Genetics, and the Society for Maternal-Fetal Medicine. Practice bulletin no. 163: screening for fetal aneuploidy. Obstet Gynecol 2016;127:e12337.

10. Gil MM, Accurti V, Santacruz B, Plana MN, Nicolaides KH. Analysis of cell-free DNA in maternal blood in screening for aneuploidies: updated meta-analysis. Ultrasound Obstet Gynecol 2017;50:302-14.

11. Gil MM, Quezada MS, Revello R, Akolekar R, Nicolaides KH. Analysis of cell-free DNA in maternal blood in screening for fetal aneuploidies: updated meta-analysis. Ultrasound Obstet Gynecol 2015;45:249-66.

12. Musci TJ, Fairbrother G, Batey A, Bruursema J, Struble C, Song K. Noninvasive prenatal testing with cell-free DNA: US physician attitudes toward implementation in clinical practice. Prenat Diagn 2013;33: 424-8.

13. Sachs A, Blanchard L, Buchanan A, Norwitz E, Bianchi DW. Recommended pre-test counseling points for noninvasive prenatal testing using cell-free DNA: a 2015 perspective. Prenat Diagn 2015;35:96871.

14. Filoche SK, Lawton B, Beard A, Stone P. Views of the obstetric profession on non-invasive prenatal testing in Aotearoa New Zealand: a national survey. Aust N Z J Obstet Gynaecol 2017;57:617-23.

15. World Health Organization. Global health observatory data, density of physicians. [http://www.who.int/gho/health_workforce/physicians_ density/en]

16. Ahn HS, Seol HJ, Lim JE, Hong SH, Lee SY, Park MI, et al. Estimates of induced abortion in South Korea: health facilities survey. J Obstet Gynaecol Res 2012;38:324-8. 